\title{
Negative thermodiffusion of polymers and colloids in solvent mixtures
}

\author{
Berend-Jan de Gans, Rio Kita, and Beate Müller \\ Max Planck Institut für Polymerforschung, Ackermannweg 10, D-55128 Mainz, Germany \\ Simone Wiegand ${ }^{\text {a) }}$ \\ Max Planck Institut für Polymerforschung, Ackermannweg 10, D-55128 Mainz, Germany \\ and Forschungszentrum Jülich GmbH, Institut für Festkörperforschung-Weiche Materie, \\ D-52428 Jülich, Germany
}

(Received 9 September 2002; accepted 3 February 2003)

\begin{abstract}
Results on thermodiffusion of poly(ethylene oxide) and colloidal boehmite ( $\gamma$-AlOOH) rods in ethanol/water mixtures are presented. Data were obtained using thermal diffusion forced Rayleigh scattering. The sign of the Soret coefficient of the boehmite rods changes from positive to negative with increasing water content, i.e., at sufficiently high water content the colloidal particles move to higher temperatures. The sign of the Soret coefficient of the poly(ethylene oxide) in ethanol/water mixtures is negative, i.e., the poly(ethylene oxide) molecules move to higher temperatures, whereas in pure water the sign is positive. To our knowledge this is the first time that a sign change has been observed for polymers in solution. The analysis of the static light scattering on poly(ethylene oxide) allows the determination of the preferentially solvating solvent. In the investigated concentration range the preferentially solvating solvent is ethanol, in spite of being the poorer solvent for poly(ethylene oxide). (C) 2003 American Institute of Physics. [DOI: 10.1063/1.1563601]
\end{abstract}

\section{INTRODUCTION}

Thermal diffusion or the Ludwig-Soret effect is the transport of mass due to a temperature gradient. In polymer solutions and colloidal suspensions partial demixing may occur, as the heavy polymer molecules or the colloids migrate to lower temperatures. In equilibrium the two oppositely directed fluxes due to thermal and ordinary diffusion cancel. The magnitude of the Ludwig-Soret effect is characterized by the Soret coefficient $S_{\mathrm{T}}$. Although a generally accepted theoretical framework for the Ludwig-Soret effect in fluids is still lacking, there is some qualitative understanding of the underlying principles. In particular, it was shown for colloidal suspensions that thermal diffusion strongly depends on the composition of the solute-solvent interface. ${ }^{1}$

Additional effects may arise when considering polymers or colloids in a mixture of solvents subjected to a temperature gradient. Due to thermal diffusion, a solvent composition gradient may build up. If the polymer under consideration has a different affinity for the different solvents, the polymer molecules will experience an affinity gradient in addition to the temperature gradient. Rue and Schimpf ${ }^{2}$ studied polystyrene in mixtures of good and bad solvents. They indeed found an enhanced $S_{\mathrm{T}}$ if the better solvent moved to the cold wall, and a diminished $S_{\mathrm{T}}$ in the opposite case. Van Asten et $\mathrm{l}^{3}{ }^{3}$ studied thermal diffusion of polystyrene in mixtures of the good solvent tetrahydrofuran and the thetasolvent cyclohexane. They found that the Soret coefficient was largely unaffected by the addition of cyclohexane. This was explained by preferential solvation of the polystyrene molecules by tetrahydrofuran independent of the bulk solvent composition. Therefore, the solute-solvent interface

${ }^{a)}$ Electronic mail: s.wiegand@fz-juelich.de does not depend on the latter, and the thermodiffusive behavior does not change either.

When investigating thermal diffusion of rodlike boehmite colloids $(\gamma-\mathrm{AlOOH})$ in ethanol/water mixtures, we observed the unusual phenomenon of colloids moving to higher temperatures, which is the opposite of what is usually found. By convention, the sign of the flux due to thermodiffusion is positive when the corresponding compound is moving to lower temperatures. ${ }^{4}$ As this system suffered from a number of disadvantages (see Sec. III A 2), we looked for a simpler polymeric system, and discovered that poly(ethylene oxide) in ethanol/water mixtures showed the same effect. Negative thermodiffusion of polymers was also observed by Giglio and Vendramini, in the system poly(vinyl alcohol)/water. ${ }^{5}$ As for colloids it was observed in magnetic fluids by Turek et al. ${ }^{6}$ In liquid mixtures such as ethanol/water, a sign change of the Soret coefficient has also been observed. ${ }^{7}$

Our data were measured using a transient holographic grating technique called thermal diffusion forced Rayleigh scattering (TDFRS). Since its development in the late seventies ${ }^{8,9}$ TDFRS has established itself as a sensitive tool to study thermal as well as ordinary diffusion, and was applied successfully to solvent mixtures ${ }^{10}$ and polymers in solution. ${ }^{11}$ The principle of TDFRS is analogous to ordinary forced Rayleigh scattering: an intensity grating is created by the interference of two laser beams. By adding a small amount of absorbing dye to the sample, the intensity grating is converted into a temperature grating, which is in turn converted into a composition grating by the effect of thermal diffusion. Both the temperature and the composition grating contribute to a refractive index grating, which is read by an additional laser beam. The advantage of TDFRS lies in its high sensitivity and the small temperature differences that occur (of the order of $\mu \mathrm{K}$ ), thereby avoiding convection and 
allowing a theoretical description of the experiment using linear irreversible thermodynamics, which is presented in the next section. The rest of the paper is organized as follows:

The experiment section contains information about sample preparation, the setup used for thermal diffusion forced Rayleigh scattering, the determination of refractive index and refractive index increments, and data analysis. Finally, we present and discuss data from a systematic study of the thermodiffusive behavior of poly(ethylene oxide) in ethanol/water as a function of solvent composition. Representative data of the more complicated system boehmite/ ethanol /water are also presented.

\section{THEORY}

In this section we will review the equations underlying the TDFRS experiment. For a thorough mathematical derivation we refer to Köhler. ${ }^{12}$ The following convention will be adopted: Species "0" denotes polymer, "1" denotes water, and " 2 " ethanol. For polymer one may also read colloid.

In principle there are three processes that have to be taken into account. First there is the diffusion of heat, which in our experiment occurs on a time scale of the order of $10^{-4}$ s. Second, in a ternary system consisting of a polymer in a mixture of solvents two different diffusive processes are expected to occur, opposing thermal diffusion. Only in the absence of cross diffusion, that is, the occurrence of a flux of the one component engendered by the concentration gradient of one of the other components, these two diffusive fluxes can be interpreted as the diffusion of polymer molecules and, on a much shorter time scale, the diffusion of solvent molecules. In our experiment we cannot exclude cross diffusion. In fact, the affinity gradient diffusion of Rue and Schimpf ${ }^{2}$ and the concept of diffusion of a polymer with preferentially solvating solvent shell of Van Asten $e t$ al. $^{3}$ are two examples of cross-diffusive processes. On the other hand, in our experiments only one diffusive process is seen, on a time scale of the order of $10^{-1} \mathrm{~s}$, coinciding with the time scale on which polymer diffusion is expected to occur. Therefore, in this paper we will interpret this process as such. For a thorough discussion of diffusion in multicomponent mixtures we refer to the book by Cussler. ${ }^{13}$ The fact that we only observe one diffusive process may be explained by TDFRS experiments on plain ethanol/water mixtures which have shown us that the contribution of solvent thermal diffusion to the TDFRS signal is negligible in the investigated concentration range (see Sec. IV A).

Thermal diffusion in multicomponent mixtures is discussed in the Appendix. It is shown that a ternary system can be described using three different thermal diffusion coefficients, two of which play a role when describing polymer thermal diffusion. Each of these two accounts for the thermal diffusion of the polymer against one of the two solvents. In this paper we will not try to separate the total signal into two contributions, and employ one single thermal diffusion coefficient instead.

Starting point is therefore the one-dimensional diffusion equation for the diffusion of polymer molecules in the limit of low concentrations, in the presence of a temperature gradient

$$
\frac{\partial}{\partial t} c_{0}(x, t)=D \frac{\partial^{2}}{\partial x^{2}} c_{0}(x, t)+D_{\mathrm{T}} \bar{c}_{0} \frac{\partial^{2}}{\partial x^{2}} T,
$$

where $c_{0}(x, t)$ denotes the local concentration of the polymer and $\bar{c}_{0}$ its average concentration, $T$ the temperature, $D$ the ordinary diffusion constant of the polymer, and $D_{\mathrm{T}}$ its thermal diffusion constant. A sinusoidal temperature gradient is assumed

$$
T(t)=\bar{T}+\Delta T e^{i q x} \theta(t),
$$

where $\Delta T$ denotes the amplitude of the grating and $q$ the wave vector. $\theta(t)$ denotes Heaviside's unit step function. Equation (1) can now be solved, the result being as follows:

$$
\begin{aligned}
& c_{0}(x, t)=\bar{c}_{0}+\Delta c_{0}(t) e^{i q x} \\
& \Delta c_{0}(t)=-\bar{c}_{0} \Delta T S_{\mathrm{T}}\left(1-e^{-q^{2} D t}\right),
\end{aligned}
$$

with the Soret coefficient $S_{\mathrm{T}}=D_{\mathrm{T}} / D$.

Usually the heterodyne TDFRS signal is studied, due to its higher sensitivity and its robustness against perturbations and experimental imperfections. ${ }^{12}$ The heterodyne signal intensity $\zeta_{\text {het }}(t)$ is proportional to the amplitude of the refractive index grating $\Delta n\left(T, c_{i}\right)$, which can be expanded to first order as

$$
\begin{aligned}
\zeta_{\text {het }}(t) & \propto \Delta n\left(T, c_{0}\right) \\
=\left[\left(\frac{\partial n}{\partial T}\right)_{p, c_{i}} \Delta T+\left(\frac{\partial n}{\partial c_{0}}\right)_{p, T, \mu_{j \neq 0}} \Delta c_{0}(t)\right], &
\end{aligned}
$$

where $p$ denotes the pressure and $\mu_{i}$ the chemical potential of the $i$ th component. Normalizing the total signal to the thermal signal and combining Eqs. (3) and (4), $\zeta_{\text {het }}(t)$ can now be written as

$$
\zeta_{\text {het }}(t)=1+\left(\frac{\partial n}{\partial T}\right)_{p, c_{i}}^{-1}\left(\frac{\partial n}{\partial c_{0}}\right)_{p, T, \mu_{j \neq 0}} S_{\mathrm{T}} c_{0}\left(1-e^{-q^{2} D t}\right) .
$$

The quantities $(\partial n / \partial T)_{p, c_{i}}$ and $\left(\partial n / \partial c_{0}\right)_{p, T, \mu_{j \neq 0}}$ do not follow from the TDFRS experiment and have to be determined separately. Special care must be taken with respect to the refractive index increment $\left(\partial n / \partial c_{0}\right)_{p, T, \mu_{j \neq 0}}$, as it contains an extra contribution which is due to the preferential solvation of the polymer by one of the two solvents. The refractive index increment can be written as

$$
\left(\frac{\partial n}{\partial c_{0}}\right)_{p, T, \mu_{j \neq 0}}=\left(\frac{\partial n}{\partial c_{0}}\right)_{p, T, c_{j \neq 0}}+\left(\frac{\partial c_{1}}{\partial c_{0}}\right)_{p, T, \mu_{j}}\left(\frac{\partial n}{\partial c_{1}}\right)_{p, T, c_{0}},
$$

where the second term on the right-hand side stems from preferential solvation. A composition change of the preferentially solvating solvent shell due to the imposed temperature gradient might affect $\left(\partial n / \partial c_{0}\right)_{p, T, \mu_{j \neq 0}}$. However, this is a second-order effect that enters Eq. (4) as $\left(\partial^{2} n / \partial c_{0} \partial T\right) \Delta c_{0} \Delta T$, and can therefore, in our first-order treatment, be neglected. 
Preferential solvation can be studied by means of static light scattering. ${ }^{14-16}$ The excess Rayleigh ratio $\Delta R(q)$ of a polymer in a single solvent can be written as

$$
\frac{K c}{\Delta R(q)}=M_{w}^{-1}\left(1+\frac{1}{3} q^{2} R_{G}^{2}\right)+2 A_{2} c,
$$

where $K=4 \pi^{2} n_{\text {solv }}^{2}(\partial n / \partial c)_{T}^{2} /\left(N_{\mathrm{av}} \lambda_{0}^{4}\right)$ denotes the optical constant, with $N_{\text {av }}$ Avogadro's number and $\lambda_{0}$ the wavelength of the light source used. $M_{\mathrm{w}}$ is the weight averaged molar mass of the polymer. $R_{G}$ denotes the radius of gyration of the polymer, and $A_{2}$ the second virial coefficient. When the polymer is dissolved in a binary solvent mixture, Eq. (7) can still be used, but $M_{\mathrm{w}}$ and $A_{2}$ have to be replaced by their apparent values $M_{\text {w,app }}$ and $A_{2, \text { app }}$.

A comparison of the "true" molar mass, as measured in a single solvent, with the apparent molar mass, measured in a solvent mixture, gives the extra contribution to the refractive index increment due to preferentially solvating solvent

$$
\begin{aligned}
\left(\frac{\partial c_{1}}{\partial c_{0}}\right)_{p, T, \mu_{j}}\left(\frac{\partial n}{\partial c_{1}}\right)_{p, T, c_{0}} & =\left(\frac{\partial n}{\partial c_{0}}\right)_{p, T, c_{j \neq 0}} \\
& \times\left[\left(\frac{M_{w, a p p}}{M_{w}}\right)^{1 / 2}-1\right] .
\end{aligned}
$$

If, in addition $\left(\partial n / \partial c_{1}\right)_{p, T, c_{0}}$ is known, the preferentially solvated solvent can be determined. The true second virial coefficient can be calculated via $A_{2}=A_{2, \text { app }}\left(M_{\text {w,app }} / M_{\mathrm{w}}\right)$.

\section{EXPERIMENT}

\section{A. TDFRS}

\section{Preparation of poly(ethylene oxide) samples}

Poly(ethylene oxide) (PEO) was synthesized by anionic polymerization of ethylene oxide in tetrahydrofuran at $60^{\circ} \mathrm{C}$. It was characterized using GPC $\left(M_{\mathrm{n}}=2.36\right.$ $\times 10^{5}$ gr.mol $^{-1}, M_{\mathrm{w}}=2.65 \times 10^{5}$ gr.mol ${ }^{-1}$ ). All samples contained 5.0 \pm 0.1 gr. $\mathrm{L}^{-1}$ PEO. Five different solvent mixtures were studied, the water content varying between $5 \%$ and $25 \%$ by weight. Three samples were prepared of each solvent mixture using calibrated $5 \mathrm{ml}$ flasks. Demineralized water was used as well absolute ethanol (Riedel-de Haën, HPLC grade). The water content of the ethanol was $0.11 \%$ by weight (Karl Fisher titration). PEO does not dissolve very easily in ethanol/water. Therefore, closed samples were heated carefully with a heat gun, and shaken vigorously until all PEO had dissolved. Most samples were stable over a period of weeks. However, samples containing 5\% wt water showed signs of flocculation after several days. The precipitated polymer could easily be redissolved by heating and shaking. Samples were filtered before use through a 0.45 $\mu \mathrm{m}$ PTFE-filter (Millipore). Quartz cuvettes with a pathlength of $0.2 \mathrm{~mm}$ (Hellma) were used as sample cells in the holographic experiment. Spurious amounts of quinizarin (Sigma-Aldrich) were added to achieve an optical density of $1-2 \mathrm{~cm}^{-1}$.

To check the influence of solvent thermodiffusion, some plain ethanol/water mixtures were also studied. Samples were prepared as described above.

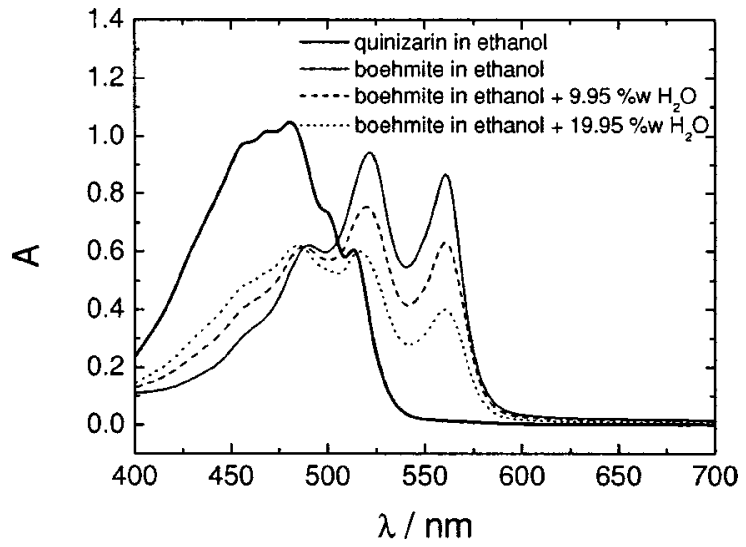

FIG. 1. Absorption spectra of suspensions of boehmite particles in ethanol/ water. Solid line: $0.00 \%$ wt $\mathrm{H}_{2} \mathrm{O}$. Dashed line: $9.95 \%$ wt $\mathrm{H}_{2} \mathrm{O}$. Dotted line: $19.95 \%$ wt $\mathrm{H}_{2} \mathrm{O}$. The quinizarin content of these three samples is identical. Compared to the absorption spectrum of quinizarin in ethanol (thick solid line) the boehmite suspensions show strong enhancement of the peak at 520 $\mathrm{nm}$, and an extra peak at $561 \mathrm{~nm}$. The strength of both absorption bands decreases with water content. The effect may be caused by adsorption of quinizarin at the surface of the boehmite particles.

As a reference, we also studied the behavior of PEO in pure water. Samples were prepared as described previously. PEO easily dissolves in water under gentle shaking. As quinizarin is insoluble in water, basantol yellow 215 (BASF) was used as dye.

\section{Preparation of boehmite samples}

A dispersion of boehmite needles in demineralized water, coded ASBIP13, was prepared according to Buining. ${ }^{17}$ Transmission electron microscopy yielded an average length of $251 \mathrm{~nm}$ (polydispersity: 40\%), and an average thickness of $8 \mathrm{~nm}$. This dispersion was transferred to absolute ethanol (Riedel-de Haën, HPLC grade), by azeotropic distillation. The dispersion was ultrasonicated (Sonorex RK514H) for 10 min, to completely redisperse the particles. The obtained stock contained 5.34 gr. $\mathrm{L}^{-1}$ boehmite. The dispersion was completely stable over several months. No formation of flocs or sediment was observed. Some quinizarin was added to the stock solution. Samples were then prepared as follows: a certain amount of the stock dispersion was diluted with demineralized water and absolute ethanol (Riedel-de Haën, HPLC grade) such that the boehmite concentration of the sample was 4.23 gr. $\mathrm{L}^{-1}$. Six different solvent mixtures were studied, the water content varying between $0 \%$ and $25 \%$ by weight. Only one sample was prepared per solvent mixture. Samples were filtered before use through a $5.0 \mu \mathrm{m}$ PTFEfilter (Millipore). As sample cells the same quartz cuvettes were used as for the poly(ethylene oxide) samples. The boehmite samples were always prepared the day before measurement.

After addition of quinizarin the color of the boehmite samples changes from orange to pink within several hours. This may be due to adsorption of quinizarin on the surface of the boehmite particles. Absorption spectra were measured using a Perkin Elmer Lambda 2 spectrophotometer and rectangular quartz cuvettes with a path length of $0.5 \mathrm{~cm}$ (Hellma). Examples are shown in Fig. 1. Compared to the 
spectrum of pure quinizarin in ethanol, the boehmite suspensions show strong enhancement of the peak at $520 \mathrm{~nm}$, and an extra peak at $561 \mathrm{~nm}$. The strength of both absorption bands decreases with water content. However, the requirements that the dye should fulfill (strong absorption at 488 $\mathrm{nm}$, negligible absorption at $632.8 \mathrm{~nm}^{13}$ ) are still met.

\section{Setup and measuring protocol}

The experimental setup has been described in detail elsewhere. ${ }^{18}$ Therefore, only a brief description will be given here. The interference grating is written by an argon-ion laser (Spectra Physics), operating at a wavelength of $\lambda_{w}$ $=488 \mathrm{~nm}$. The refractive index grating is read by a heliumneon laser (Spectra Physics), operating at $\lambda_{r}=632.8 \mathrm{~nm}$. A photomultiplier tube (Thorn Emi) measures the intensity of the diffracted laser light. The two writing beams are initially vertically polarized. A Pockels cell (Leysop) is used to rotate the polarization of one of the writing beams by $180^{\circ}$. This corresponds to a phase shift of the refractive index grating of $180^{\circ}$. Compared to switching the grating on and off, this procedure has the advantage of stronger contrast and hence stronger signal intensity.

To separate the heterodyne from the homodyne signal, we proceed as follows: Using a mirror, mounted on a piezo translator (Piezo Systeme Jena), the phase of the interference grating is shifted by $180 \mathrm{deg}$. Subtraction of the two phaseshifted signals gives the pure heterodyne signal. The piezo mirror can also be used for the phase stabilization of the interference grating. ${ }^{11}$

As quinizarin is not bleached, the experiment can be repeated until the desired signal quality is reached. In the case of the poly(ethylene oxide) samples 2000 measurements were averaged. The intensity of the argon laser beams just before hitting the sample was about $30 \mathrm{~mW}$. As for the boehmite samples, 1000 measurements were averaged at 45 $\mathrm{mW}$ laser power. Lower laser light intensities give the same results. All measurements were performed at room temperature, at a wave vector of $4.6 \times 10^{5} \mathrm{~m}^{-1}$.

\section{B. Data analysis}

Figure 2 shows typical decaying and increasing diffraction signals of TDFRS measurements. The measurement starts at $t=0$. At $t=t^{\prime}$ the phase of the interference grating is shifted over $180^{\circ}$. The data $F(t)$ can be represented by the following expression:

$$
\begin{aligned}
F(t)= & {\left[\theta(t)-\theta\left(t-t^{\prime}\right)\right] F_{1}(t) } \\
& +\left[\theta\left(t-t^{\prime}\right)-\theta\left(t-2 t^{\prime}\right)\right] F_{2}(t),
\end{aligned}
$$

where $\theta(t)$ denotes Heaviside's unit step function. $F_{1}$ and $F_{2}$ are some functions representing the data. $F_{1}$ and $F_{2}$ are then subtracted as follows:

$$
y=F_{1}(t)-F_{2}\left(t+t^{\prime}\right) .
$$

As the solute is usually polydisperse, the result is fitted to a second-order cumulant expansion

$$
y=a_{0}+a_{1} \exp \left(-a_{2} t-a_{3} t^{2}\right) .
$$

$S_{\mathrm{T}}$ and $D$ can then be determined from the fitted parameters as follows:

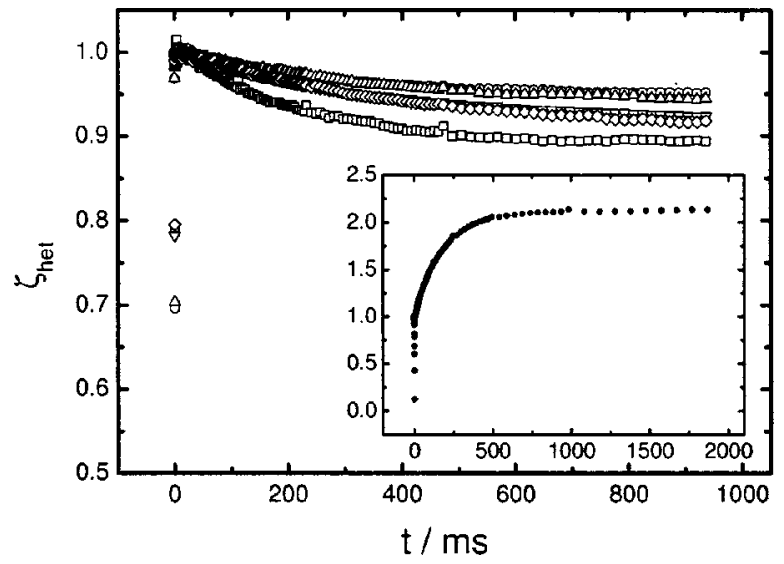

FIG. 2. Typical normalized TDFRS signals of solution of poly(ethylene oxide) in ethanol/water $[5(\bigcirc), 10(\triangle), 15(\nabla), 20(\diamond), 25(\square) \% \mathrm{wt}$ $\mathrm{H}_{2} \mathrm{O}$ ). The inset shows the raising diffraction signal of poly(ethylene oxide) in pure water.

$$
\begin{aligned}
& \left(1-\frac{2 a_{0}}{a_{1}}\right)^{-1}=\left(\frac{\partial n}{\partial T}\right)_{p, c_{i}}^{-1}\left(\frac{\partial n}{\partial c_{0}}\right)_{p, T, \mu_{j \neq 0}} c_{0} S_{\mathrm{T}}, \\
& a_{2}=q^{2} D
\end{aligned}
$$

This procedure avoids the use of step functions during fitting.

\section{Contrast factors}

The temperature derivative of the refractive index $(\partial n / \partial T)_{p, c_{j}}$ was determined at $632.8 \mathrm{~nm}$, in the temperature range 293-298 $\mathrm{K}$, using a scanning Michelson interferometer. $^{19}$ Figure 3(a) shows $(\partial n / \partial T)_{p, c_{j}}$ of the poly(ethylene oxide) samples as a function of solvent composition. The temperature derivative of the refractive index of boehmite in pure ethanol was measured as well. It was found that $(\partial n / \partial T)_{p, c_{j}}=4.05 \times 10^{-4} \mathrm{~K}^{-1}$.

Refractive index increments $\left(\partial n / \partial c_{0}\right)_{p, T, c_{j \neq 0}}$ were measured at room temperature with our own setup ${ }^{19}$ also operating at $632.8 \mathrm{~nm}$. Samples were prepared as described previously. Figure 3(b) shows $\left(\partial n / \partial c_{0}\right)_{p, T, c_{j \neq 0}}$ of the poly(ethylene oxide) samples as a function of solvent composition. It is seen that $\left(\partial n / \partial c_{0}\right)_{p, T, c_{j \neq 0}}$ does not depend strongly on solvent composition. The refractive index increment of boehmite in pure ethanol was measured as well. It was found that $\left(\partial n / \partial c_{0}\right)_{p, T}=0.1004 \mathrm{ml} \cdot \mathrm{gr}^{-1}$.

The refractive index $n$ of solutions, containing 5 gr. $\mathrm{L}^{-1}$ poly(ethylene oxide) and varying water content, were measured using an Abbé refractometer (Atago-Kübler) operating at $589 \mathrm{~nm}$ and $293 \mathrm{~K}$. The temperature was kept constant to within $0.1 \mathrm{~K}$. Samples were prepared as described previously. Figure 3(c) shows a plot of $n$ as a function of $\phi_{m, \mathrm{H}_{2} \mathrm{O}}$. $\left(\partial n / \partial c_{1}\right)_{p, T, c_{0}}$ was calculated from a fourth-order polynomial fit to the data, which is shown as the dotted line in Fig. $3(\mathrm{c})$. It is seen that when $\phi_{m, \mathrm{H}_{2} \mathrm{O}}=0.193,\left(\partial n / \partial c_{1}\right)_{p, T, c_{0}}$ is approximately zero, that is, at $\phi_{m, \mathrm{H}_{2} \mathrm{O}}=0.193$ the solvent contribution to the refractive index increment 


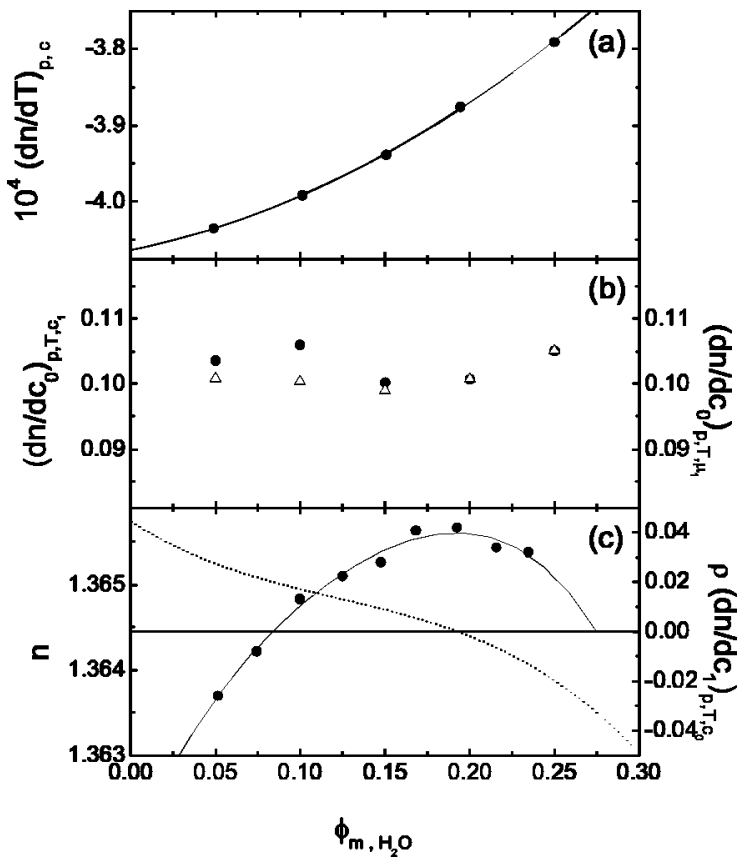

FIG. 3. (a) $(\partial n / \partial T)_{p, c_{i}}$ of the poly(ethylene oxide) samples as a function of solvent composition. The solid line is a second-order polynomial fit to the data. (b) : $\left(\partial n / \partial c_{0}\right)_{p, c_{1}}$ of the poly(ethylene oxide) samples as a function of solvent composition. $\left(\partial n / \partial c_{0}\right)_{p, c_{1}}$ depends only weakly on solvent composition. $\triangle:\left(\partial n / \partial c_{0}\right)_{p, \mu_{1}}$. (c) Refractive index $n$ of poly(ethylene oxide) samples at constant polymer concentration as a function of solvent composition. The solid line is a fourth-order polynomial fit to the data. The dotted line represents the derivative $\rho\left(\partial n / \partial c_{1}\right)_{p, c_{0}}$. At $\phi_{m, \mathrm{H}_{2} \mathrm{O}}=0.193$, $\rho\left(\partial n / \partial c_{1}\right)_{p, c_{0}}=0$, that is, at this composition preferential solvation does not contribute to the total refractive index increment $\left(\partial n / \partial c_{0}\right)_{p, \mu_{1}}$ [see Eq. (6)].

$\left(\partial n / \partial c_{0}\right)_{p, T, \mu_{j \neq 0}}$ can be neglected. This was used to measure the true molar mass of our poly(ethylene oxide).

\section{Static light scattering}

Poly(ethylene oxide) samples for static light scattering were prepared as described previously. Cylindrical quartz cuvettes with a diameter of $20 \mathrm{~mm}$ (Hellma) were used as sample cells. All cuvettes were made dustfree by rinsing them with freshly distilled acetone for at least 15 min using an acetone fountain. Static light scattering measurements were carried out using an ALV 5000E instrument. All measurements were performed at room temperature using a Krypton laser (Spectra Physics) of wavelength $\lambda_{0}$ in vacuum equal to $647.1 \mathrm{~nm}$ at $150 \mathrm{~mW}$ output. Data were taken every $5^{\circ}$ ranging from $30^{\circ}-150^{\circ}$. Data were corrected for solvent background and converted into Rayleigh ratios as follows:

$$
\Delta R(q)=\frac{I_{\text {solution }}(q)-I_{\text {solv }}}{I_{\text {ref }}}\left(\frac{n_{\text {solv }}}{n_{\text {ref }}}\right)^{2} R_{\text {ref }},
$$

where $I_{\text {solution }}, I_{\text {solv }}$, and $I_{\text {ref }}$ denote the scattered intensities of solution, solvent, and reference respectively. $n_{\text {solv }}$ and $n_{\text {ref }}$ denote the refractive index of solvent and reference. Toluene was used as a reference, the Rayleigh ratio of which was taken to be $1.27 \times 10^{-5} \mathrm{~cm}^{-1}$.

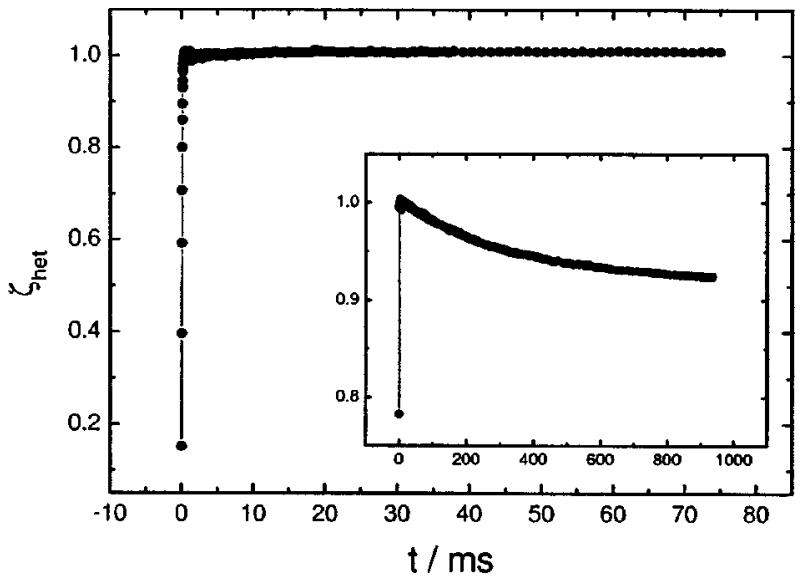

FIG. 4. TDFRS signal of an ethanol/water mixture containing $17.88 \% \mathrm{wt}$ water. The solid line connects the data points. It is seen that the concentration contribution to the total signal is negligible. For comparison the inset shows the normalized diffraction signal for PEO in a ethanol/water mixture ( $15 \%$ wt $\mathrm{H}_{2} \mathrm{O}$ ).

\section{RESULTS AND DISCUSSION}

\section{A. Ethanol/water mixtures}

Figure 4 shows a typical result of a TDFRS experiment on an ethanol/water mixture. The slow contribution due to thermal diffusion is small compared to the fast contribution due to temperature, about $1 \%$ of the total signal. Therefore, the assumption that the contribution of solvent thermal diffusion to the total heterodyne signal is negligible, made in deriving Eq. (5), is justified. In a study of the thermal lens effect in ethanol-water mixtures, Arnaud and Georges arrived at the same conclusion. ${ }^{20}$ However, at mass fractions of water higher than $25 \%$ the neglect of solvent thermal diffusion is no longer justified.

To interpret our results on polymers and colloids, knowledge about the direction of the thermodiffusion flux in the solvent mixture is required. A recent international study, in which a number of different measuring methods were compared, showed clearly that ethanol diffuses to higher temperatures, although uncertainties of the values were large because of the smallness of the effect. ${ }^{21}$

\section{B. Boehmite samples}

Figure 5 shows typical results for TDFRS measurements on boehmite dispersions. Figure 5(a) shows the result of a measurement on a boehmite dispersion, containing $4.06 \%$ water by weight. The direction of the thermodiffusive flux can now be found as follows: Figure 5(a) shows that the thermal contribution and the concentration contribution to the refractive index grating are in phase. $(\partial n / \partial T)_{p, c_{i}}$ is negative as always. It seems reasonable to assume that $\left(\partial n / \partial c_{0}\right)_{p, T, \mu_{j \neq 0}}$ is positive, as the rotationally averaged refractive index of boehmite is $n_{d}^{20}=1.65$, the refractive index of water is $n_{d}^{20}=1.333$, and the refractive index of ethanol is $n_{d}^{20}=1.362 .{ }^{22}$ Therefore, $\Delta T$ positive implies a negative $\Delta c$ [see Eq. (4)], which is the same as saying that the thermodiffusive flux is directed to lower temperatures. Figure 5(b) shows the result of a measurement on a boehmite dispersion 

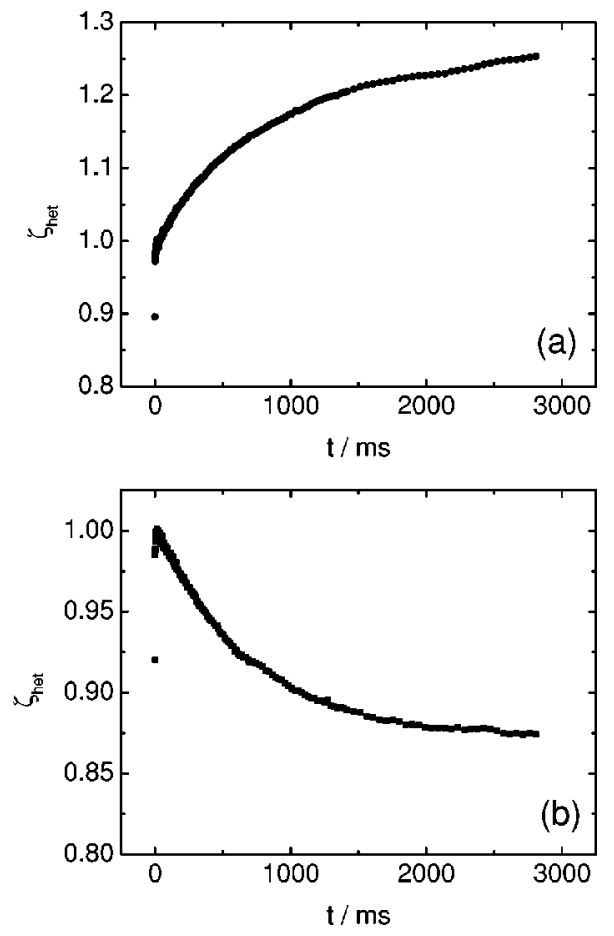

FIG. 5. (a) TDFRS signal of a boehmite dispersion containing $4.06 \% \mathrm{wt}$ water. The temperature and concentration contribution to the refractive index grating are in phase. The boehmite particles move to lower temperatures. (b) TDFRS signal of a boehmite dispersion containing 5.52\% wt water. In this case, the temperature and concentration contribution to the refractive index grating are $180^{\circ}$ out of phase. This implies that with increasing water content the boehmite particles start moving to higher temperatures.

containing $5.52 \%$ water by weight. Thermal and concentration contributions to the refractive index grating are clearly $180^{\circ}$ out of phase. This implies that the thermodiffusive flux is in this case directed to higher temperatures. Further increase of the water content does not change this picture.

The sign change of the thermodiffusive flux within a narrow composition range was qualitatively reproducible. Unfortunately, it proved to be very difficult to quantitatively reproduce the location of the transition. Moreover, because of the adsorption of quinizarin on the surface of the boehmite particles, it is not unlikely that upon irradiation by the laser beams the local temperature around the particles is higher than average. The transition phenomenon seems to be sufficiently interesting to deserve further study. Unfortunately the experimental difficulties kept us from doing so. A detailed experimental study would require a major change of the experiment by using, e.g., a laser wavelength in the absorption range of water so that no dye needs to be added.

The diffusion coefficients that follow from fitting Eq. (11) to the data are shown in Fig. 6. It is seen that the diffusion coefficient of the boehmite rods in ethanol/water has a maximum. Using the values for $(\partial n / \partial T)_{p, c_{i}}$ and $\left(\partial n / \partial c_{0}\right)_{p, T}$ mentioned in Sec. III C, the Soret coefficient $S_{\mathrm{T}}$ and the thermal diffusion coefficient $D_{\mathrm{T}}$ of boehmite rods in pure ethanol were calculated. It was found that $S_{\mathrm{T}}=0.14 \mathrm{~K}^{-1}$, and $D_{\mathrm{T}}$ $=7.2 \times 10^{-9} \mathrm{~cm}^{2} \cdot \mathrm{s}^{-1} \cdot \mathrm{K}^{-1}$.

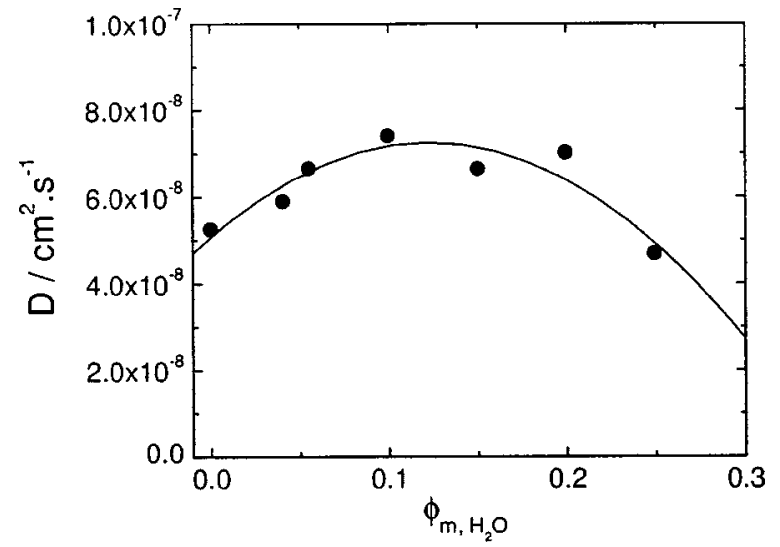

FIG. 6. Diffusion constant $D$ of boehmite particles as a function of water content. The solid line is a quadratic fit to the data.

\section{Poly(ethylene oxide) samples}

Figure 2 shows the normalized heterodyne diffraction signal of a series of TDFRS measurements on poly(ethylene oxide) samples in several ethanol/water mixtures. Samples contained $5 \%$ to $25 \% \mathrm{H}_{2} \mathrm{O}$ by weight. The direction of the thermodiffusive flux can be found as before, taking into account that $(\partial n / \partial T)_{p, c_{i}}$ is negative and $\left(\partial n / \partial c_{0}\right)_{p, T, \mu_{j \neq 0}}$ is positive. It turns out that the flux is directed to higher temperatures. This result is independent of solvent composition. The inset of Fig. 2 shows the result of a TDFRS measurement on poly(ethylene oxide) in pure water. In this case $(\partial n / \partial T)_{p, c}$ is negative and $(\partial n / \partial c)_{p, T}$ is positive, implying that the flux is directed to lower temperatures, in agreement with results obtained by Kirkland using thermal field flow fractionation. ${ }^{23}$ Pure ethanol at room temperature is a flocculant for poly(ethylene oxide); therefore, experiments to determine the direction of the thermodiffusive flux in pure ethanol are difficult.

Figure 7(a) shows the diffusion constant $D$, calculated according to Eq. (13). Figure 7(b) shows the thermal diffusion constant $D_{\mathrm{T}}$. It is seen that the error in $D_{\mathrm{T}}$ is considerable, about $20 \%$.

The results of the static light scattering measurements on poly(ethylene oxide) in ethanol/water as a function of solvent composition are summarized in Table I. Shown are the apparent molar mass $M_{w \text {,app }}$, the radius of gyration $R_{G}$, and the second virial coefficient $A_{2}$. The molar mass, measured at $\phi_{m, \mathrm{H}_{2} \mathrm{O}}=0.20$, and the molar mass measured with GPC (see Sec. III A 1), agree within 5\%, which is reasonable. The second virial coefficients $A_{2}$ are positive, indicating that ethanol/water mixtures are good solvents for poly(ethylene oxide). In Fig. 3(b) the refractive index increments $(\partial n / \partial c)_{p, T, \mu_{i}}$ are shown. It is seen that the contribution of preferential solvation to $(\partial n / \partial c)_{p, T, \mu_{i}}$ is limited to $6 \%$ or less.

From the apparent molar masses and Eq. (8), it follows that the contribution of preferential solvation to the refractive index increment $\left(\partial c_{1} / \partial c_{0}\right)_{p, T, \mu_{j}}\left(\partial n / \partial c_{1}\right)_{p, T, c_{0}}$ is always negative. As $\left(\partial n / \partial c_{1}\right)_{p, T, c_{0}}$ is positive when $\phi_{m, \mathrm{H}_{2} \mathrm{O}}<0.20$ [see Fig. 3(c)], it follows that $\left(\partial c_{1} / \partial c_{0}\right)_{p, T, \mu_{j}}$ is negative as 


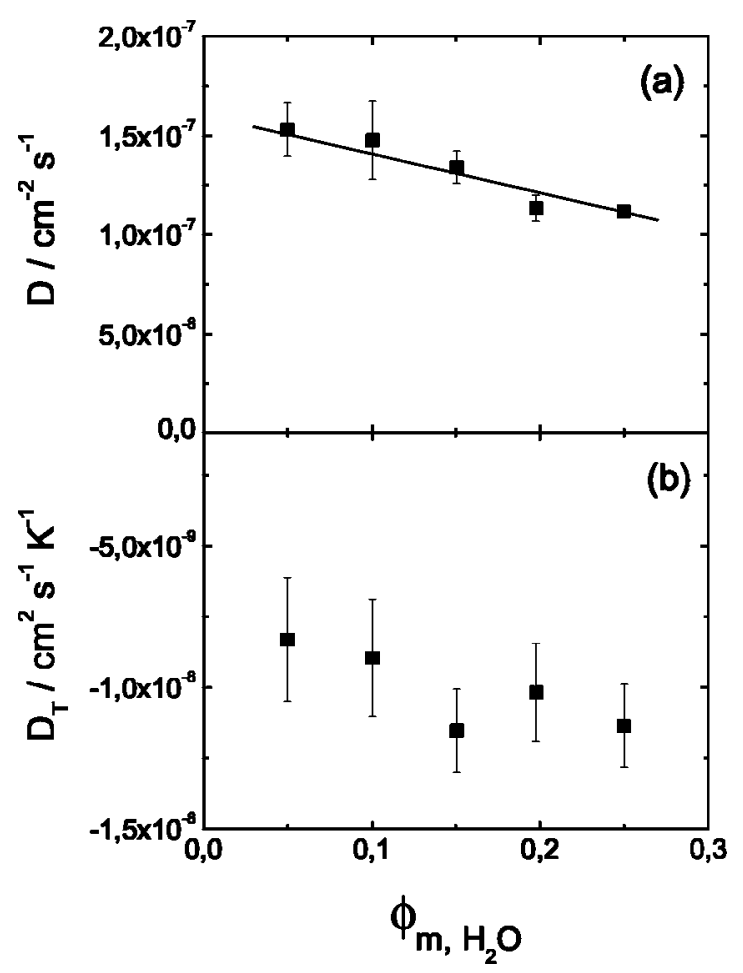

FIG. 7. (a) Diffusion constant $D$ of poly(ethylene oxide) as a function of water content. The solid line is a linear fit to the data. (b) Thermal diffusion constant $D_{\mathrm{T}}$ as a function of water content. It is seen that the error in the data is considerable.

well. This implies that poly(ethylene oxide) is preferentially solvated by the component 2 (ethanol), though ethanol is the poorer solvent. However, it is known that preferential solvation cannot be understood on the basis of the solvation properties of the individual solvents, and that the size of the solvent molecules relative to each other and the affinity of the solvents for each other also plays a role. ${ }^{16}$ Preferential solvation by the poorer solvent has been reported in literature, for example in the system poly(methyl methacrylate)/ benzene/methanol, ${ }^{15}$ where methanol is the poorer solvent.

In the binary solvent mixture ethanol/water at ethanol content higher than $\phi_{m, \mathrm{EtOH}}=0.289$, ethanol moves to higher temperatures, whereas water moves to lower temperatures. The poly(ethylene oxide) coils are preferentially solvated by ethanol. Therefore, the thermodiffusive behavior of poly(ethylene oxide) in ethanol/water can be understood

TABLE I. Apparent molar mass $M_{w \text {,app }}$, radius of gyration $R_{G}$, and true second virial coefficient $A_{2}$ of the poly(ethylene oxide) used as a function of solvent composition. The value of $M_{w}$ at $\phi_{m, \mathrm{H}_{2} \mathrm{O}}=20 \%$ was taken to be the true molecular weight. From the values of the second virial coefficient $A_{2}$, it follows that ethanol/water mixtures are good solvents for poly(ethylene oxide).

\begin{tabular}{rccc}
\hline \hline $\begin{array}{c}\phi_{m, \mathrm{H}_{2} \mathrm{O}} / \\
\% \mathrm{wt}\end{array}$ & $\begin{array}{c}M_{w, \mathrm{app}^{\prime}} \mathrm{kg} \cdot \mathrm{mol}^{-1} \\
5.00\end{array}$ & $\begin{array}{c}R_{G} / \\
\mathrm{nm}\end{array}$ & $\begin{array}{c}A_{2} / \\
\mathrm{mol} \cdot \mathrm{cm}^{3} \cdot \rho^{-2}\end{array}$ \\
\hline 10.02 & 239 & 26.7 & 11.4 \\
15.00 & 226 & 23.8 & 12.9 \\
20.00 & 246 & 25.0 & 12.6 \\
24.99 & 252 & 31.7 & 14.0 \\
\hline \hline
\end{tabular}

qualitatively by the model of Van Asten, ${ }^{3}$ which states that thermodiffusion of a polymer molecule in a solvent mixture is determined by the properties of the interface, preferentially solvated solvent-bulk solvent. However, the explanation in terms of an affinity gradient which was used by Rue and Schimpf, ${ }^{2}$ may explain our results as well. However, it should be realized that what matters is not the affinity of the polymer for each solvent separately, but rather how preferential solvation changes with solvent composition. It would therefore be interesting to study thermodiffusion in systems like poly(methyl methacrylate) in acetonitril/1-chlorobutane, as the preferentially solvating solvent changes with increasing acetonitril content from acetonitril to 1-chlorobutane. ${ }^{16}$ Another interesting system would be poly(ethylene oxide) in ethanol/water at low ethanol content, as it has been established that the sign of the Soret coefficient changes at $\phi_{m, \mathrm{EtOH}}=0.289 .^{8}$

Recent calculations carried out on the system poly(ethylene oxide) in ethanol/water have yielded a deeper understanding of the thermodiffusive behavior of polymers in solvent mixtures. These calculations agree qualitatively with the experimental results, i.e., the bad solvent ethanol solvates the poly(ethylene oxide), which moves to higher temperatures. Moreover, it was found that polymer thermodiffusion in a solvent mixture depends in a complicated way on the interactions of the three components simultaneously, and cannot be understood on the basis of simple models as proposed by Van Asten ${ }^{3}$ and Rue and Schimpf. ${ }^{2}$ The results of these calculations will be published elsewhere. ${ }^{24,25}$

\section{CONCLUSIONS}

We present data on the thermodiffusive behavior of polymers and colloidal particles in ethanol/water mixtures. Data were measured using thermal diffusion forced Rayleigh scattering (TDFRS). The colloidal particles are ionically stabilized boehmite $(\gamma-\mathrm{AlOOH})$ rods. The sign of the Soret coefficient of this system changes from positive to negative with increasing water content, i.e., at sufficiently high water content the colloidal particles move to higher temperatures. The polymer used in this study is poly(ethylene oxide). The sign of the Soret coefficient was negative for all investigated ethanol/water mixtures, whereas in pure water PEO the sign is positive. To our knowledge this is the first time that negative and positive thermodiffusion has been observed for the same polymer.

The system poly(ethylene oxide)/ethanol/water was also investigated using static light scattering. This yielded the contribution of preferentially solvating solvent to the TDFRS signal, and therefore allowed the correct determination of the Soret coefficient. It was found that at high ethanol concentrations the preferentially solvating solvent is ethanol, in spite of this being the poorer solvent for poly(ethylene oxide). This unusual solvation behavior illustrates the complexity of the interactions between the three components, which may also account for the observed sign change of the Soret coefficient in the polymer solvent mixtures. As in ethanol/ water mixtures in the investigated concentration range ethanol moves to higher temperatures as well, the behavior of poly(ethylene oxide) can be understood by assuming a shell 
of preferentially solvating ethanol around the poly(ethylene oxide) coil, which determines the direction in which the coil moves. Alternatively, a poly(ethylene oxide) coil can be looked upon as if moving in a affinity gradient, established by thermal diffusion within the solvent mixture. The polymer molecule will then move into the direction of the thermodynamically favored preferentially solvating solvent shell composition.

\section{ACKNOWLEDGMENTS}

The authors are indebted to Jean Platten, who shared his insights in the definition of the Soret coefficient of multicomponent systems. We would like to thank Abbas Firoozabadi for sending his publications on multicomponent mixtures. We thank Thomas Wagner for the synthesis of the poly(ethylene oxide). We gratefully acknowledge the donation of basantol yellow 215 by BASF (Ludwigshafen). We acknowledge Petra Räder for carrying out the Karl Fisher titration. We thank Christine Rosenauer for her help in carrying out the apparent molar mass determinations. We want to thank Jutta Luettmer-Strathmann and Florian MüllerPlathe for helpful discussions. Finally, we would like to thank Elizabeth Lupton for correcting the manuscript.

\section{APPENDIX: SORET COEFFICIENT IN MULTICOMPONENT MIXTURES}

In our data analysis a single thermal diffusion coefficient was used to describe the behavior of polymer molecules in a binary solvent mixture, subjected to a temperature gradient. However, it seems obvious that for the full description of thermodiffusion in a multicomponent mixture more than one coefficient is needed. In the literature, a number of conflicting approaches exists for the definition of thermal diffusion coefficients in multicomponent mixtures. ${ }^{26-29}$ In this Appendix we will develop an approach to the definition of thermal diffusion coefficients in multicomponent mixtures, which, in the case of a ternary system, is identical to the definition of Larre et al. ${ }^{29}$

First, consider the simple case of a binary mixture of particles 1 and 2, subjected to a temperature gradient. Due to thermal diffusion we have two fluxes, $J_{1}$ and $J_{2}$. Assuming continuity, the sum of fluxes should be zero, that is $J_{1}+J_{2}$ $=0$. Each of the two fluxes should be proportional to the temperature gradient $\partial T / \partial x$ and to the concentration of particles $c_{\mathrm{i}}$. However, the definition of the thermal diffusion coefficient as $J_{1}=-D_{\mathrm{T}, \mathrm{c}} c_{\mathrm{i}} /(\partial T / \partial x)$ has the disadvantage that for each of the two components a different thermal diffusion coefficient is needed. This problem can be circumvented by using the following definition:

$$
J=-D_{\mathrm{T}} c_{1} c_{2} \frac{\partial T}{\partial x}=-D_{\mathrm{T}} c_{1}\left(1-c_{1}\right) \frac{\partial T}{\partial x},
$$

allowing the description of a binary system using one single thermal diffusion coefficient.

The total thermal diffusion flux of a single component in a ternary mixture is the sum of a series of fluxes of the type $J_{\mathrm{ij}}$, describing the exchange of one component against the other due to their different thermal diffusive behavior

$$
\begin{aligned}
& J_{1}=J_{12}+J_{13}=-D_{\mathrm{T}, 12} c_{1} c_{2}-D_{\mathrm{T}, 13} c_{1} c_{3} \frac{\partial T}{\partial x}, \\
& J_{2}=-J_{12}+J_{23}=D_{\mathrm{T}, 12} c_{1} c_{2}-D_{\mathrm{T}, 23} c_{2} c_{3} \frac{\partial T}{\partial x}, \\
& J_{3}=-J_{13}-J_{23}=D_{\mathrm{T}, 13} c_{1} c_{3}+D_{\mathrm{T}, 23} c_{2} c_{3} \frac{\partial T}{\partial x} .
\end{aligned}
$$

As in the binary case it makes sense to define that each flux $J_{\mathrm{ij}}$ is proportional to the product $c_{\mathrm{i}} c_{\mathrm{j}}$, thereby limiting the number of independent thermal diffusion coefficients needed to describe the ternary system to three. This result is identical to that of Larre et al. ${ }^{29}$ It is important to notice that if one of the three concentrations is set equal to zero we recover the binary case, as we should.

If the system contains $N$ components, the thermal diffusion flux of component $i$ can be written as

$$
J_{\mathrm{i}}=\sum_{i \neq j}^{N} J_{\mathrm{ij}}=-\sum_{i \neq j}^{N} D_{\mathrm{T}, \mathrm{ij}} c_{\mathrm{i}} c_{\mathrm{j}} \frac{\partial T}{\partial x} .
$$

For the full description of such a system $\frac{1}{2} N(N-1)$ different thermal diffusion coefficients are needed. Furthermore, it is important to notice that this definition guarantees that the sum of all fluxes $\sum_{i=1}^{N} J_{\mathrm{i}}=0$.

In the approach of Kempers ${ }^{26}$ the thermal diffusion flux of a component $i$ is written as

$$
J_{\mathrm{i}}=-D_{\mathrm{T}, \mathrm{i}} c_{i}\left(1-c_{i}\right) \frac{\partial T}{\partial x} .
$$

This definition seems unnatural, as the requirement that the sum of all fluxes be zero does not follow directly from the definition of the fluxes, but merely imposes a restriction on the values and signs of the thermal diffusion coefficients.

In our analysis of the data, the polymer thermal diffusion flux is approximated as $J_{0}=-D_{\mathrm{T}, 0} c_{0}(\partial T / \partial x)$. The thusdefined polymer thermal diffusion coefficient is therefore a weighted sum of two thermal diffusion coefficients

$$
D_{\mathrm{T}, 0}=c_{1} D_{\mathrm{T}, 01}+c_{2} D_{\mathrm{T}, 02} .
$$

Unfortunately, we cannot recover the values of $D_{\mathrm{T}, 01}$ and $D_{\mathrm{T}, 02}$.

${ }^{1}$ P. M. Shiundu and J. C. Giddings, J. Chromatogr., A 715, 117 (1995).

${ }^{2}$ C. A. Rue and M. E. Schimpf, Anal. Chem. 66, 4054 (1994).

${ }^{3}$ A. C. van Asten, W. T. Kok, R. Tijsen, and H. Poppe, J. Polym. Sci., Part B: Polym. Phys. 34, 283 (1996).

${ }^{4}$ W. Köhler and S. Wiegand, Thermal Non-Equilibrium Phenomena in Fluid Mixtures (LNP 584, Springer, Berlin, 2002).

${ }^{5}$ M. Giglio and A. Vendramini, Phys. Rev. Lett. 38, 26 (1977).

${ }^{6}$ I. Turek, J. Štelina, C. Musil, M. Timko, P. Kopčanský, M. Koneracká, and L. Tomčo, J. Magn. Magn. Mater. 201, 167 (1999).

${ }^{7}$ P. Kolodner, H. Williams, and C. Moe, J. Chem. Phys. 88, 6512 (1988)

${ }^{8}$ T. Thyagarajan and L. Lallemand, Opt. Commun. 26, 54 (1978).

${ }^{9}$ P. Pohl, Phys. Lett. 77A, 53 (1980).

${ }^{10}$ W. Köhler and B. Müller, J. Chem. Phys. 103, 4367 (1995).

${ }^{11}$ W. Köhler, J. Chem. Phys. 98, 660 (1993).

${ }^{12}$ W. Köhler and R. Schäfer, Adv. Polym. Sci. 151, 1 (2000).

${ }^{13}$ E. L. Cussler, Diffusion-Mass Transfer in Fluid Systems (Cambridge University Press, Cambridge, 1985), p. 194.

${ }^{14}$ J. M. G. Cowie and I. J. McEwen, Macromolecules 7, 291 (1974).

${ }^{15}$ I. Katime and C. Strazielle, Makromol. Chem. 178, 2295 (1977). 
${ }^{16}$ R. M. Masegosa, M. G. Prolongo, I. Hernández-Fuentes, and A. Horta, Macromolecules 17, 1181 (1984).

${ }^{17}$ P. A. Buining, C. Pathmamanoharan, J. B. H. Jansen, and H. N. W. Lekkerkerker, J. Am. Ceram. Soc. 74, 1303 (1991).

${ }^{18}$ A. Perronace, C. Leppla, F. Leroy, B. Rousseau, and S. Wiegand, J. Chem. Phys. 116, 3718 (2002).

${ }^{19}$ A. Becker, W. Köhler, and B. Müller, Ber. Bunsenges. Phys. Chem. 99, 600 (1995).

${ }^{20}$ N. Arnaud and J. Georges, Spectrochim. Acta, Part A 57, 1295 (2001)

${ }^{21}$ J. K. Platten, M. M. Bou-Ali, P. Costeséque, J. F. Dutrieux, W. Köhler, C. Leppla, S. Wiegand, and G. Wittko, J. Chem. Phys. (to be published).
${ }^{22}$ The CRC Handbook of Chemistry and Physics, edited by D. R. Lide (CRC, Boca Raton, 1995).

${ }^{23}$ J. J. Kirkland and W. W. Yau, J. Chromatogr. 353, 95 (1986).

${ }^{24}$ B. J. de Gans, S. Wiegand, and J. Luettmer-Strathmann, Phys. Rev. Lett. (to be published).

${ }^{25}$ J. Luettmer-Strathmann (unpublished).

${ }^{26}$ L. Kempers, J. Chem. Phys. 90, 6541 (1989).

${ }^{27}$ K. Ghorayeb and A. Firoozabadi, AIChE J. 46, 883 (2000).

${ }^{28}$ S. V. Vaerenbergh and J. Legros, Entropie 198/199, 77 (1996).

${ }^{29}$ J. P. Larre, J. Platten, and G. Chavepeyer, Int. J. Heat Mass Transf. 40, 545 (1997). 